JUNE 1991, PAGES 201-213

\title{
INEQUALITIES ASSOCIATED WITH STANDING WAVE SOLUTIONS OF A HYPERBOLIC EQUATION
}

\author{
BY \\ W. A. DAY \\ Hertford College, Oxford, England
}

1. Introduction. The objective of this paper is to draw attention to certain inequalities associated with a function $u$ which is a standing wave solution (separable solution) of the hyperbolic equation

$$
\frac{\partial}{\partial x}\left(\tau(x) \frac{\partial u}{\partial x}\right)+f(x, t)=\rho(x) \frac{\partial^{2} u}{\partial t^{2}} \quad(a<x<b,-\infty<t<\infty),
$$

and which satisfies the boundary conditions

$$
u(a, t)=u(b, t)=0 \quad(-\infty<t<\infty) .
$$

If the coefficient $\tau$ is constant on $[a, b]$, the differential equation describes the transverse deflection $u$ of a string, of mass density $\rho$, which is stretched to a tension $\tau$ and whose ends $x=a$ and $x=b$ are held fixed. The string is subjected to a transverse load of density $f$. In what follows we shall not need to suppose $\tau$ to be constant.

It will emerge later that some of our results carry over to a fourth-order boundary value problem which describes the static transverse deflection of a loaded elastic beam, and to higher-order problems of a special type.

We are concerned with what happens when $f$ is harmonic in its dependence upon the time, with frequency $\omega / 2 \pi$, and when $u$ is the harmonic steady-state response to $f$. Thus, it is supposed that

$$
f(x, t)=f(x) \cos \omega t, \quad u(x, t)=u(x) \cos \omega t .
$$

On substituting into the hyperbolic equation and the boundary conditions, we arrive at the self-adjoint boundary value problem

$$
\frac{d}{d x}\left(\tau(x) \frac{d u}{d x}\right)+\omega^{2} \rho(x) u+f(x)=0, \quad u(a)=u(b)=0 .
$$

Now let the string be subject to the load $f$, let its ends be fixed, and, in addition, let the point $x=c$ be held undisplaced, where $a<c<b$ but $c$ is otherwise arbitrary. In these circumstances, the deflection coincides, on the subinterval $[a, c]$, 
with $v$, and, on the subinterval $[c, b]$, with $w$, where $v$ and $w$ are determined by the respective boundary value problems

$$
\begin{array}{cl}
\frac{d}{d x}\left(\tau(x) \frac{d v}{d x}\right)+\omega^{2} \rho(x) v+f(x)=0, & v(a)=v(c)=0, \\
\frac{d}{d x}\left(\tau(x) \frac{d w}{d x}\right)+\omega^{2} \rho(x) w+f(x)=0, & w(c)=w(b)=0 .
\end{array}
$$

Our purpose is to relate the deflection which occurs when just the ends $x=a$ and $x=b$ are fixed to the deflection which occurs when the point $x=c$ is fixed as well; in other words, our purpose is to connect $u$ with $v$ and $w$.

In fact, we shall do more than this. Let the set $\left\{a_{0}, a_{1}, \ldots, a_{n}\right\}$ be any partition of the interval $[a, b]$, i.e., let

$$
a=a_{0}<a_{1}<\cdots<a_{n-1}<a_{n}=b,
$$

and, for each $i=1, \ldots, n$, let $u_{i}$ be the function which is defined on the subinterval $\left[a_{i-1}, a_{i}\right]$ as the solution of the boundary value problem

$$
\frac{d}{d x}\left(\tau(x) \frac{d u_{i}}{d x}\right)+\omega^{2} \rho(x) u_{i}+f(x)=0, \quad u_{i}\left(a_{i-1}\right)=u_{i}\left(a_{i}\right)=0 .
$$

What is envisaged here is that the ends $x=a$ and $x=b$ are held undisplaced and so are the internal points $x=a_{i}(i=1, \ldots, n-1)$; once again, we aim to connect $u$ with $u_{1}, \ldots, u_{n}$.

In what follows it will be necessary to restrict the forcing frequency $\omega / 2 \pi$ to be less than the fundamental frequency of the unloaded string.

It will be supposed throughout that $\tau, \rho$, and $f$, are positive and continuous on $[a, b]$. The requirement that $f$ be of constant sign is a crucial one. No further restrictions will be placed upon $f$ except in deriving Eqs. (2.12) and (3.4) which refer to specific functional forms of $f$.

Although the results are different, the methods of this paper have something in common with those of my paper [1], and of a related paper by S. Friedland [2], in that they rely upon detailed knowledge of kernels associated with Green's functions of ordinary differential operators.

2. Behaviour when $\omega=0$. Our conclusions are easiest to understand in the static case $(\omega=0)$ and, since the conclusions for the dynamic case $(\omega>0)$ depend upon those for the static case, it is convenient to start by dealing with what happens when $\omega=0$.

When $\omega=0$, the boundary value problems (1.1) and (1.2) reduce, respectively, to the problems

$$
\begin{array}{cc}
\frac{d}{d x}\left(\tau(x) \frac{d u}{d x}\right)+f(x)=0, & u(a)=u(b)=0, \\
\frac{d}{d x}\left(\tau(x) \frac{d u_{i}}{d x}\right)+f(x)=0, & u_{i}\left(a_{i-1}\right)=u_{i}\left(a_{i}\right)=0 .
\end{array}
$$

Each of these problems has one and only one solution. 
Because $\tau$ and $f$ are positive, it must be that

$$
u(x)>u_{i}(x)>0 \quad\left(a_{i-1}<x<a_{i}, \quad i=1, \ldots, n\right),
$$

as an elementary argument establishes.

It is clear from (2.3) that the area under the graph of $u / \tau$ exceeds the weighted mean of each $u_{i} / \tau$, i.e.,

$$
\int_{a}^{b} \frac{u}{\tau} d x>\int_{a_{i-1}}^{a_{i}} \frac{u_{i}}{\tau} d x \quad(i=1, \ldots, n) .
$$

It is not immediately clear, though it is in fact true, that the weighted mean of $u$, with weight $1 / \tau$, exceeds the weighted mean of each $u_{i}$, i.e.,

$$
\left(\int_{a}^{b} \frac{1}{\tau} d x\right)^{-1} \int_{a}^{b} \frac{u}{\tau} d x>\left(\int_{a_{i-1}}^{a_{i}} \frac{1}{\tau} d x\right)^{-1} \int_{a_{i-1}}^{a_{i}} \frac{u_{i}}{\tau} d x \quad(i=1, \ldots, n) .
$$

Indeed, more is true: if $\omega=0$, the weighted mean of $u$ exceeds the sum of the weighted means of $u_{1}, \ldots, u_{n}$, i.e.,

$$
\left(\int_{a}^{b} \frac{1}{\tau} d x\right)^{-1} \int_{a}^{b} \frac{u}{\tau} d x>\sum_{i=1}^{n}\left(\int_{a_{i-1}}^{a_{i}} \frac{1}{\tau} d x\right)^{-1} \int_{a_{i-1}}^{a_{i}} \frac{u_{i}}{\tau} d x
$$

In the case of a stretched string, $\tau$ is constant and (2.4) reduces to the inequality

$$
\frac{1}{b-a} \int_{a}^{b} u d x>\sum_{i=1}^{n} \frac{1}{a_{i}-a_{i-1}} \int_{a_{i-1}}^{a_{i}} u_{i} d x \text {. }
$$

The key to the proof of $(2.4)$ is the identity

$$
\begin{aligned}
(D-C) & (X-A)(B-X) \\
= & (B-A)(X-C)(D-X) \\
& +((B-A)-(D-C))\left(X+\frac{A D-B C}{(B-A)-(D-C)}\right)^{2} \\
& +\frac{(B-A)(D-C)(C-A)(B-D)}{(B-A)-(D-C)},
\end{aligned}
$$

from which it follows that if $A<C<D<B$ then

$$
\frac{(X-A)(B-X)}{B-A}>\frac{(X-C)(D-X)}{D-C}
$$

for all $X$.

Next, we observe that, as an elementary argument establishes,

$$
\begin{gathered}
\int_{a}^{b} \frac{u}{\tau} d x=\int_{a}^{b} K f d x \\
\int_{a_{i-1}}^{a_{i}} \frac{u_{i}}{\tau} d x=\int_{a_{i-1}}^{a_{i}} K_{i} f d x \quad(i=1, \ldots, n),
\end{gathered}
$$


where $K, K_{1}, \ldots, K_{n}$ are the kernels

$$
\begin{gathered}
K(x)=\frac{1}{2} \int_{a}^{x} \frac{d y}{\tau(y)} \int_{x}^{b} \frac{d y}{\tau(y)}, \\
K_{i}(x)=\frac{1}{2} \int_{a_{i-1}}^{x} \frac{d y}{\tau(y)} \int_{x}^{a_{i}} \frac{d y}{\tau(y)} \quad(i=1, \ldots, n) .
\end{gathered}
$$

However, if in (2.5) we choose

$$
X=\int_{a}^{x} \frac{d y}{\tau(y)}
$$

and

$$
A=0, \quad B=\int_{a}^{b} \frac{d y}{\tau(y)}, \quad C=\int_{a}^{a_{i-1}} \frac{d y}{\tau(y)}, \quad D=\int_{a}^{a_{i}} \frac{d y}{\tau(y)}
$$

we see that

$$
\begin{aligned}
& \left(\int_{a}^{b} \frac{d y}{\tau(y)}\right)^{-1} \int_{a}^{x} \frac{d y}{\tau(y)} \int_{x}^{b} \frac{d y}{\tau(y)} \\
& >\left(\int_{a_{i-1}}^{a_{i}} \frac{d y}{\tau(y)}\right)^{-1} \int_{a_{i-1}}^{x} \frac{d y}{\tau(y)} \int_{x}^{a_{i}} \frac{d y}{\tau(y)} \quad(i=1, \ldots, n),
\end{aligned}
$$

and, therefore, that

$$
\left(\int_{a}^{b} \frac{d x}{\tau}\right)^{-1} K>\left(\int_{a_{i-1}}^{a_{i}} \frac{d x}{\tau}\right)^{-1} K_{i} \quad(i=1, \ldots, n) .
$$

Hence

$$
\begin{aligned}
\left(\int_{a}^{b} \frac{d x}{\tau}\right)^{-1} \int_{a}^{b} K f d x & =\left(\int_{a}^{b} \frac{d x}{\tau}\right)^{-1} \sum_{i=1}^{n} \int_{a_{i-1}}^{a_{i}} K f d x \\
& >\sum_{i=1}^{n}\left(\int_{a_{i-1}}^{a_{i}} \frac{d x}{\tau}\right)^{-1} \int_{a_{i-1}}^{a_{i}} K_{i} f d x,
\end{aligned}
$$

and, in view of (2.6) and (2.7), this last inequality is equivalent to the advertised inequality (2.4).

Since

$$
\int_{a}^{b} \frac{1}{\tau} d x>\int_{a_{i-1}}^{a_{i}} \frac{1}{\tau} d x \quad(i=1, \ldots, n)
$$

it is evident from (2.4) that

$$
\int_{a}^{b} \frac{u}{\tau} d x>\sum_{i=1}^{n} \int_{a_{i-1}}^{a_{i}} \frac{u_{i}}{\tau} d x
$$

i.e., that the area under the graph of $u / \tau$ exceeds the sum of the areas under the graphs of $u_{1} / \tau, \ldots, u_{n} / \tau$. This fact is also an obvious consequence of the inequalities (2.3). The inequality (2.4), though, implies a stronger statement about areas: if 
$\omega=0$ the areas under the graphs of $u / \tau, u_{1} / \tau, \ldots, u_{n} / \tau$ satisfy the inequality

$$
\left(\int_{a}^{b} \frac{u}{\tau} d x\right)^{1 / 2}>\sum_{i=1}^{n}\left(\int_{a_{i-1}}^{a_{i}} \frac{u_{i}}{\tau} d x\right)^{1 / 2} .
$$

To verify (2.8) we need only combine (2.4) with the Cauchy-Schwarz inequality to find that

$$
\begin{aligned}
& {\left[\sum_{i=1}^{n}\left(\int_{a_{i-1}}^{a_{i}} \frac{u_{i}}{\tau} d x\right)^{1 / 2}\right]^{2}} \\
& \quad \leq \sum_{i=1}^{n} \int_{a_{i-1}}^{a_{i}} \frac{d x}{\tau} \cdot \sum_{i=1}^{n}\left(\int_{a_{i-1}}^{a_{i}} \frac{d x}{\tau}\right)^{-1} \int_{a_{i-1}}^{a_{i}} \frac{u_{i}}{\tau} d x \\
& \quad=\int_{a}^{b} \frac{d x}{\tau} \cdot \sum_{i=1}^{n}\left(\int_{a_{i-1}}^{a_{i}} \frac{d x}{\tau}\right)^{-1} \int_{a_{i-1}}^{a_{i}} \frac{u_{i}}{\tau} d x \\
& \quad<\int_{a}^{b} \frac{d x}{\tau}\left(\int_{a}^{b} \frac{d x}{\tau}\right)^{-1} \int_{a}^{b} \frac{u}{\tau} d x=\int_{a}^{b} \frac{u}{\tau} d x,
\end{aligned}
$$

and, on taking the square root of each side, we arrive at (2.8).

The inequality (2.8) is actually the best of its kind, in the sense that it cannot be replaced by an inequality

$$
\left(\int_{a}^{b} \frac{u}{\tau} d x\right)^{\theta}>\sum_{i=1}^{n}\left(\int_{a_{i-1}}^{a_{i}} \frac{u_{i}}{\tau} d x\right)^{\theta},
$$

with an exponent $\theta<\frac{1}{2}$ which is independent of $f$.

We prove this by showing that if $\varepsilon$ is any positive number, no matter how small, it is possible to choose a partition of $[a, b]$, and to choose $\tau$ and $f$ (the choice of $f$ depending upon $\varepsilon$ ) in such a way that $\tau$ and $f$ are positive and continuous on $[a, b]$ and the inequality (2.9) holds only if $\theta>\frac{1}{2}-\varepsilon$.

It is understood here, as it is throughout this section, that $\omega=0$. In order to verify the statement, let the partition be the set $\left\{a_{0}, a_{1}, a_{2}\right\}$, where $a_{0}=a, a_{1}=$ $(a+b) / 2, a_{2}=b$, let $\tau \equiv 1$, and let the load be

$$
f(x)=\left(x-\frac{a+b}{2}\right)^{2 N}+\xi_{N}\left(\frac{b-a}{2}\right)^{2 N} \quad(a \leq x \leq b),
$$

where $N$ is a large positive integer which will depend upon $\varepsilon$, and $\left\{\xi_{N}\right\}$ is any sequence of positive numbers for which $\xi_{N}=o\left(N^{-2}\right)$ as $N \rightarrow \infty$. For this particular $f$, the bulk of the load is concentrated near the ends $x=a$ and $x=b$. In these circumstances,

$$
\begin{aligned}
\int_{a}^{b} \frac{u}{\tau} d x & =\frac{1}{2} \int_{a}^{b}(x-a)(b-x) f(x) d x \\
& =\left(\frac{b-a}{2}\right)^{2 N+3}\left[\frac{2}{(2 N+1)(2 N+3)}+\frac{2}{3} \xi_{N}\right],
\end{aligned}
$$




$$
\begin{aligned}
\int_{a_{0}}^{a_{1}} \frac{u_{1}}{\tau} d x & =\frac{1}{2} \int_{a}^{(a+b) / 2}(x-a)\left(\frac{a+b}{2}-x\right) f(x) d x \\
& =\left(\frac{b-a}{2}\right)^{2 N+3}\left[\frac{1}{2(2 N+2)(2 N+3)}+\frac{1}{12} \xi_{N}\right], \\
\int_{a_{1}}^{a_{2}} \frac{u_{2}}{\tau} d x & =\frac{1}{2} \int_{(a+b) / 2}^{b}\left(x-\frac{a+b}{2}\right)(b-x) f(x) d x \\
& =\left(\frac{b-a}{2}\right)^{2 N+3}\left[\frac{1}{2(2 N+2)(2 N+3)}+\frac{1}{12} \xi_{N}\right] .
\end{aligned}
$$

Thus (2.9) holds only if

$$
\left[\frac{2}{(2 N+1)(2 N+3)}+\frac{2}{3} \xi_{N}\right]^{\theta}>2\left[\frac{1}{2(2 N+2)(2 N+3)}+\frac{1}{12} \xi_{N}\right]^{\theta},
$$

i.e., only if

$$
\theta \log \eta_{N}>\log 2
$$

where

$$
\eta_{N}=\left[\frac{2}{(2 N+1)(2 N+3)}+\frac{2}{3} \xi_{N}\right] /\left[\frac{1}{2(2 N+2)(2 N+3)}+\frac{1}{12} \xi_{n}\right] .
$$

The order relation $\xi_{N}=o\left(N^{-2}\right)$ ensures that $\eta_{N} \rightarrow 4$ as $N \rightarrow \infty$ and, since we may suppose that $\varepsilon<\frac{1}{2}$, we can arrange that $\log \eta_{N}<\log 4 /(1-2 \varepsilon)$ by choosing $N$ sufficiently large. When $N$ is chosen in this way, (2.10) tells us that (2.9) holds only if

as was claimed.

$$
\theta>(1-2 \varepsilon) \frac{\log 2}{\log 4}=\frac{1}{2}-\varepsilon,
$$

This last conclusion notwithstanding, there are special choices of $f$ which ensure that (2.9) holds with an exponent $\theta<\frac{1}{2}$. Indeed, there are circumstances in which the cube roots of the areas under the graphs of $u / \tau, u_{1} / \tau, \ldots, u_{n} / \tau$, satisfy an addition law: if the load

$$
f(x)=\frac{p}{\tau(x)} \quad(a \leq x \leq b),
$$

where $p$ is a constant, then

$$
\left(\int_{a}^{b} \frac{u}{\tau} d x\right)^{1 / 3}=\sum_{i=1}^{n}\left(\int_{a_{i-1}}^{a_{i}} \frac{u_{i}}{\tau} d x\right)^{1 / 3}
$$

To prove (2.12) we need only note that, as direct integration reveals,

$$
\int_{a}^{b} \frac{u}{\tau} d x=\frac{1}{2} p \int_{a}^{b}\left(\int_{a}^{x} \frac{d y}{\tau(y)} \int_{x}^{b} \frac{d y}{\tau(y)}\right) \frac{1}{\tau(x)} d x=\frac{1}{12} p\left(\int_{a}^{b} \frac{d x}{\tau(x)}\right)^{3}
$$

and similarly,

$$
\int_{a_{i-1}}^{a_{i}} \frac{u_{i}}{\tau} d x=\frac{1}{12} p\left(\int_{a_{t-1}}^{a_{i}} \frac{d x}{\tau(x)}\right)^{3} \quad(i=1, \ldots, n) .
$$


Since

$$
\int_{a}^{b} \frac{d x}{\tau(x)}=\sum_{i=1}^{n} \int_{a_{i-1}}^{a_{i}} \frac{d x}{\tau(x)}
$$

(2.12) is correct.

It might be asked whether, for suitable choices of $f$, there are other addition laws

$$
\left(\int_{a}^{b} \frac{u}{\tau} d x\right)^{\theta}=\sum_{i=1}^{n}\left(\int_{a_{i-1}}^{a_{i}} \frac{u_{i}}{\tau} d x\right)^{\theta}
$$

with exponents $\theta \neq \frac{1}{3}$.

We turn to showing that such cannot be the case; to do so we note that $(2.13)$ is equivalent to the assertion that

$$
\begin{aligned}
& {\left[\int_{a}^{b}\left(\int_{a}^{x} \frac{d y}{\tau(y)} \int_{x}^{b} \frac{d y}{\tau(y)}\right) f(x) d x\right]^{\theta}} \\
& \quad=\sum_{i=1}^{n}\left[\int_{a_{i-1}}^{a_{i}}\left(\int_{a_{i-1}}^{x} \frac{d y}{\tau(y)} \int_{x}^{a_{i}} \frac{d y}{\tau(y)}\right) f(x) d x\right]^{\theta} .
\end{aligned}
$$

What we shall show is that: the equation

$$
\begin{aligned}
& {\left[\int_{\alpha}^{\beta}\left(\int_{\alpha}^{x} \frac{d y}{\tau(y)} \int_{x}^{\beta} \frac{d y}{\tau(y)}\right) f(x) d x\right]^{\theta}} \\
& \quad=\sum_{i=1}^{n}\left[\int_{\alpha_{i-1}}^{\alpha_{i}}\left(\int_{\alpha_{i-1}}^{x} \frac{d y}{\tau(y)} \int_{x}^{\alpha_{i}} \frac{d y}{\tau(y)}\right) f(x) d x\right]^{\theta}
\end{aligned}
$$

cannot be valid for every subinterval $[\alpha, \beta] \subset[a, b]$, and for all partitions $\left\{\alpha_{0}, \alpha_{1}\right.$, $\left.\ldots, \alpha_{n}\right\}$ of the subinterval, unless $\theta=\frac{1}{3}$.

To prove this let $c$ be any point in $a<c<b$, and let $\varepsilon$ be any number in $0<\varepsilon<1$. Since $\tau$ and $f$ are positive and continuous we can choose $\delta>0$ so that

$$
\left.\begin{array}{l}
(1-\varepsilon) \tau(c)<\tau(x)<(1+\varepsilon) \tau(c), \\
(1-\varepsilon) f(c)<f(x)<(1+\varepsilon) f(c),
\end{array}\right\}
$$

for $a<c-\delta<x<c+\delta<b$. Now let us take $\alpha=c-\delta$ and $\beta=c+\delta$. The three points $\{c-\delta, c, c+\delta\}$ constitute a partition of the subinterval $[c-\delta, c+\delta]$ and, if $(2.14)$ if valid,

$$
\begin{aligned}
{\left[\int_{c-\delta}^{c+\delta}\right.} & \left.\left(\int_{c-\delta}^{x} \frac{d y}{\tau(y)} \int_{x}^{c+\delta} \frac{d y}{\tau(y)}\right) f(x) d x\right]^{\theta} \\
= & {\left[\int_{c-\delta}^{c}\left(\int_{c-\delta}^{x} \frac{d y}{\tau(y)} \int_{x}^{c} \frac{d y}{\tau(y)}\right) f(x) d x\right]^{\theta} } \\
& +\left[\int_{c}^{c+\delta}\left(\int_{c}^{x} \frac{d y}{\tau(y)} \int_{x}^{c+\delta} \frac{d y}{\tau(y)}\right) f(x) d x\right]^{\theta} .
\end{aligned}
$$


The estimates (2.15) imply that

$$
\begin{aligned}
\frac{4}{3} \frac{\delta^{3}(1-\varepsilon) f(c)}{(1+\varepsilon)^{2} \tau(c)^{2}} & <\int_{c-\delta}^{c+\delta}\left(\int_{c-\delta}^{x} \frac{d y}{\tau(y)} \int_{x}^{c+\delta} \frac{d y}{\tau(y)}\right) f(x) d x \\
& <\frac{4}{3} \frac{\delta^{3}(1+\varepsilon) f(c)}{(1-\varepsilon)^{2} \tau(c)^{2}} \\
\frac{1}{6} \frac{\delta^{3}(1-\varepsilon) f(c)}{(1+\varepsilon)^{2} \tau(c)^{2}} & <\int_{c-\delta}^{c}\left(\int_{c-\delta}^{x} \frac{d y}{\tau(y)} \int_{x}^{c} \frac{d y}{\tau(y)}\right) f(x) d x \\
& <\frac{1}{6} \frac{\delta^{3}(1+\varepsilon) f(c)}{(1-\varepsilon)^{2} \tau(c)^{2}} \\
\frac{1}{6} \frac{\delta^{3}(1-\varepsilon) f(c)}{(1+\varepsilon)^{2} \tau(c)^{2}} & <\int_{c}^{c+\delta}\left(\int_{c}^{x} \frac{d y}{\tau(y)} \int_{x}^{c+\delta} \frac{d y}{\tau(y)}\right) f(x) d x \\
& <\frac{1}{6} \frac{\delta^{3}(1+\varepsilon) f(c)}{(1-\varepsilon)^{2} \tau(c)^{2}},
\end{aligned}
$$

and since $\tau(c), f(c)$, and $\delta$, are positive it must be that

$$
\begin{aligned}
& {\left[\frac{4}{3} \frac{(1+\varepsilon)}{(1-\varepsilon)^{2}}\right]^{\theta}>2\left[\frac{1}{6} \frac{(1-\varepsilon)}{(1+\varepsilon)^{2}}\right]^{\theta},} \\
& {\left[\frac{4}{3} \frac{(1-\varepsilon)}{(1+\varepsilon)^{2}}\right]^{\theta}<2\left[\frac{1}{6} \frac{(1+\varepsilon)}{(1-\varepsilon)^{2}}\right]^{\theta},}
\end{aligned}
$$

and, hence,

$$
\left[8\left(\frac{1+\varepsilon}{1-\varepsilon}\right)^{3}\right]^{\theta}>2>\left[8\left(\frac{1-\varepsilon}{1+\varepsilon}\right)^{3}\right]^{\theta}
$$

for every $\varepsilon$ in $0<\varepsilon<1$. On letting $\varepsilon \rightarrow 0$ we deduce that $8^{\theta}=2$ and, hence, that $\theta=\frac{1}{3}$, as required.

3. Behaviour when $\omega>0$. We return now to the more general boundary value problems (1.1) and (1.2). The forcing frequency will be restricted by the requirement that $0<\omega<\omega_{0}$, where $\omega_{0}^{2}$ is the least eigenvalue associated with the SturmLiouville problem

$$
\frac{d}{d x}\left(\tau(x) \frac{d \psi}{d x}\right)+\omega^{2} \rho(x) \psi=0, \quad \psi(a)=\psi(b)=0 .
$$

We start by showing that if $0<\omega<\omega_{0}$ there is a $\phi$ which is positive on $[a, b]$ and which satisfies the homogeneous equation

$$
\frac{d}{d x}\left(\tau(x) \frac{d \phi}{d x}\right)+\omega^{2} \rho(x) \phi=0 .
$$

It is known (Hartman [3, p. 351, Corollary 6.1]) that the existence of such a $\phi$ is equivalent to the disconjugacy of the operator

on $[a, b]$.

$$
\frac{d}{d x}\left(\tau(x) \frac{d}{d x}\right)+\omega^{2} \rho(x)
$$


Let us attempt to define $\phi$ as the solution of the boundary value problem

$$
\frac{d}{d x}\left(\tau(x) \frac{d \phi}{d x}\right)+\omega^{2} \rho(x) \phi=0, \quad \phi(a)=\phi(b)=1 .
$$

By virtue of the fact that (3.1) can be replaced by an integral equation to which the Fredholm Alternative applies, both the existence and the uniqueness of $\phi$ follow once it is known that the problem

$$
\frac{d}{d x}\left(\tau(x) \frac{d \chi}{d x}\right)+\omega^{2} \rho(x) \chi=0, \quad \chi(a)=\chi(b)=0,
$$

has no solution other than $\chi \equiv 0$. This, though, is certainly the case if $0<\omega<\omega_{0}$ and, hence, $\phi$ is well defined by (3.1).

Next, we show that $\phi>0$ throughout the interval $[a, b]$. To do so let $\psi_{0}$ be an eigenfunction corresponding to the least eigenvalue $\omega_{0}^{2}$. Then

$$
\frac{d}{d x}\left(\tau(x) \frac{d \psi_{0}}{d x}\right)+\omega_{0}^{2} \rho(x) \psi_{0}=0, \quad \psi_{0}(a)=\psi_{0}(b)=0,
$$

and, as is well known, $\psi_{0}$ has no zero in the open interval $[a, b]$. If it were not the case that $\phi>0$ in $[a, b]$ there would exist a subinterval $[c, d] \subset[a, b]$ such that $\phi(c)=\phi(d)=0$ and $\phi<0$ in $(c, d)$. In view of the inequality $\omega^{2} \rho<\omega_{0}^{2} \rho$, Sturm's Separation Theorem ([3, p. 335)] would then tell us that the eigenfunction $\psi_{0}$ has a zero in $(c, d)$, which is false. Thus $\phi$ must be positive throughout $[a, b]$.

It is now clear that, if $0<\omega<\omega_{0}$, each of the boundary value problems (1.1) and (1.2) has one and only one solution.

For, if we make the substitutions

$$
u=\phi U, \quad u_{i}=\phi U_{i} \quad(i=1, \ldots, n),
$$

we find that (1.1) and (1.2) are replaced by the problems

$$
\begin{array}{ll}
\frac{d}{d x}\left(\phi^{2} \tau \frac{d U}{d x}\right)+\phi f=0, & U(a)=U(b)=0, \\
\frac{d}{d x}\left(\phi^{2} \tau \frac{d U_{i}}{d x}\right)+\phi f=0, & U_{i}\left(a_{i-1}\right)=U_{i}\left(a_{i}\right)=0,
\end{array}
$$

respectively, and each of these has one and only one solution.

Each of these problems is of the type already discussed in Sec. 2, but with $\tau$ replaced by $\phi^{2} \tau$ and with $f$ replaced by $\phi f$. Thus, if we note that

$$
\int_{a}^{b} \frac{U}{\phi^{2} \tau} d x=\int_{a}^{b} \frac{u}{\phi^{3} \tau} d x
$$

and so forth, we can read off from (2.4) and (2.8) that, if $0<\omega<\omega_{0}$, the inequalities

$$
\left(\int_{a}^{b} \frac{d x}{\phi^{2} \tau}\right)^{-1} \int_{a}^{b} \frac{u}{\phi^{3} \tau} d x>\sum_{i=1}^{n}\left(\int_{a_{i-1}}^{a_{i}} \frac{d x}{\phi^{2} \tau}\right)^{-1} \int_{a_{i-1}}^{a_{i}} \frac{u_{i}}{\phi^{3} \tau} d x
$$




$$
\left(\int_{a}^{b} \frac{u}{\phi^{3} \tau} d x\right)^{1 / 2}>\sum_{i=1}^{n}\left(\int_{a_{i-1}}^{a_{i}} \frac{u_{i}}{\phi^{3} \tau} d x\right)^{1 / 2},
$$

must hold.

While (3.3) is concerned with areas under the graphs of $u / \phi^{3} \tau, u_{1} / \phi^{3} \tau, \ldots$, $u_{n} / \phi^{3} \tau$, the inequality (3.2) is concerned with weighted means of $u / \phi, u_{1} / \phi, \ldots$, $u_{n} / \phi$, the weight being $1 / \phi^{2} \tau$. Thus (3.2) and (3.3) differ from their counterparts (2.4) and (2.8) through factors involving $\phi$, where $\phi$ itself depends upon the forcing frequency $\omega / 2 \pi$. When $\omega=0$ we can take $\phi \equiv 1$, and thereby we recover (2.4) and (2.8). When $\tau$ and $\rho$ are constant on $[a, b]$,

$$
\omega_{0}=\frac{\pi}{b-a}\left(\frac{\tau}{\rho}\right)^{1 / 2}
$$

and we can take, for example,

$$
\phi(x)=\cos \left\{\omega\left(\frac{\rho}{\tau}\right)^{1 / 2}\left(x-\frac{1}{2}(a+b)\right)\right\} .
$$

There is also a counterpart to the cube-root addition law (2.12) which says that, if $0<\omega<\omega_{0}$ and if the load

$$
f(x)=\frac{p}{\phi^{3}(x) \tau(x)} \quad(a \leq x \leq b),
$$

where $p$ is a constant, then

$$
\left(\int_{a}^{b} \frac{u}{\phi^{3} \tau} d x\right)^{1 / 3}=\sum_{i=1}^{n}\left(\int_{a_{t-1}}^{a_{i}} \frac{u_{i}}{\phi^{3} \tau} d x\right)^{1 / 3} .
$$

4. A fourth-order problem. Consider an elastic beam, of unit flexural rigidity, which is subject to a transverse load of density $f$ and whose ends $x=a$ and $x=b$ are clamped to the $x$-axis. The deflection $u$, assumed to be small, is the solution of the boundary value problem

$$
\left.\begin{array}{l}
-\frac{d^{4} u}{d x^{4}}+f(x)=0, \\
u(a)=\frac{d u}{d x}(a)=u(b)=\frac{d u}{d x}(b)=0 .
\end{array}\right\}
$$

The load $f$ is required to be positive and continuous on $[a, b]$.

If an interior point $x=c$ is clamped to the $x$-axis, the deflection coincides on $[a, c]$ with $v$, and on $[c, b]$ with $w$, where $v$ and $w$ are determined by the boundary value problems

$$
\left.\begin{array}{l}
-\frac{d^{4} v}{d x^{4}}+f(x)=0, \\
v(a)=\frac{d v}{d x}(a)=v(c)=\frac{d v}{d x}(c)=0,
\end{array}\right\}
$$




$$
\left.\begin{array}{l}
-\frac{d^{4} w}{d x^{4}}+f(x)=0, \\
w(c)=\frac{d w}{d x}(c)=w(b)=\frac{d w}{d x}(b)=0 .
\end{array}\right\}
$$

Our purpose is to connect $u$ with $v$ and $w$ or, more generally, with $u_{1}, \ldots, u_{n}$, where $u_{i}(i=1, \ldots, n)$ is the solution of the boundary value problem

$$
\left.\begin{array}{l}
-\frac{d^{4} u_{i}}{d x^{4}}+f(x)=0, \\
u_{i}\left(a_{i-1}\right)=\frac{d u_{i}}{d x}\left(a_{i-1}\right)=u_{i}\left(a_{i}\right)=\frac{d u_{i}}{d x}\left(a_{i}\right)=0,
\end{array}\right\}
$$

and $\left\{a_{0}, a_{1}, \ldots, a_{n}\right\}$ is any partition of the interval $[a, b]$.

Repeated integration by parts leads to the formulae

$$
\begin{aligned}
\int_{a}^{b} u d x & =\int_{a}^{b} L f d x \\
\int_{a_{i-1}}^{a_{i}} u_{i} d x & =\int_{a_{i-1}}^{a_{i}} L_{i} f d x \quad(i=1, \ldots, n),
\end{aligned}
$$

in which

$$
\begin{gathered}
L(x)=\frac{1}{24}(x-a)^{2}(b-x)^{2}, \\
L_{i}(x)=\frac{1}{24}\left(x-a_{i-1}\right)^{2}\left(a_{i}-x\right)^{2} \quad(i=1, \ldots, n) .
\end{gathered}
$$

The inequality (2.5), with $X=x, A=a, B=b, C=a_{i-1}, D=a_{i}$, tells us that

$$
\frac{(x-a)(b-x)}{b-a}>\frac{\left(x-a_{i-1}\right)\left(a_{i}-x\right)}{a_{i}-a_{i-1}}
$$

and, on squaring both sides, we see that

$$
\frac{L(x)}{(b-a)^{2}}>\frac{L_{i}(x)}{\left(a_{i}-a_{i-1}\right)^{2}} \quad\left(a_{i-1} \leq x \leq a_{i} ; i=1, \ldots, n\right) .
$$

Thus

$$
\begin{aligned}
\frac{1}{(b-a)^{2}} \int_{a}^{b} L f d x & =\frac{1}{(b-a)^{2}} \sum_{i=1}^{n} \int_{a_{i-1}}^{a_{i}} L f d x \\
& >\sum_{i=1}^{n} \frac{1}{\left(a_{i}-a_{i-1}\right)^{2}} \int_{a_{i-1}}^{a_{i}} L_{i} f d x,
\end{aligned}
$$

and we conclude, with the help of (4.1), that the inequality

$$
\frac{1}{(b-a)^{2}} \int_{a}^{b} u d x>\sum_{i=1}^{n} \frac{1}{\left(a_{i}-a_{i-1}\right)^{2}} \int_{a_{i-1}}^{a_{i}} u_{i} d x
$$

is valid. 
The inequality (4.2) is the counterpart, in the context of our fourth-order problem, of the inequality (2.4).

There is a counterpart to (2.8) which says that the areas under the graphs of $u, u_{1}, \ldots, u_{n}$ satisfy the inequality

$$
\left(\int_{a}^{b} u d x\right)^{1 / 3}>\sum_{i=1}^{n}\left(\int_{a_{i-1}}^{a_{i}} u_{i} d x\right)^{1 / 3} .
$$

To prove this we need only appeal to (4.2) and to Hölder's inequality to find that

$$
\begin{aligned}
& {\left[\sum_{i=1}^{n}\left(\int_{a_{i-1}}^{a_{i}} u_{i} d x\right)^{1 / 3}\right]^{3}} \\
& \quad \leq\left[\sum_{i=1}^{n}\left(a_{i}-a_{i-1}\right)\right]^{2} \sum_{i=1}^{n} \frac{1}{\left(a_{i}-a_{i-1}\right)^{2}} \int_{a_{i-1}}^{a_{i}} u_{i} d x \\
& \quad=(b-a)^{2} \sum_{i=1}^{n} \frac{1}{\left(a_{i}-a_{i-1}\right)^{2}} \int_{a_{i-1}}^{a_{i}} u_{i} d x<\int_{a}^{b} u d x .
\end{aligned}
$$

Direct integration also reveals that when the load is uniformly distributed, i.e., when $f$ is identically constant, the areas satisfy the addition law

$$
\left(\int_{a}^{b} u d x\right)^{1 / 5}=\sum_{i=1}^{n}\left(\int_{a_{i-1}}^{a_{i}} u_{i} d x\right)^{1 / 5} .
$$

5. Higher-order problems. The results of the preceding section can be extended in routine fashion to boundary value problems, of even order, which are of the form:

$$
\left.\begin{array}{l}
\frac{d^{2 N} u}{d x^{2 N}}=(-1)^{N} f(x), \\
u=\frac{d u}{d x}=\cdots=\frac{d^{N-1}}{d x^{N-1}} u=0 \quad \text { at } x=a \text { and } x=b .
\end{array}\right\}
$$

As before, $f$ is required to be positive and continuous on $[a, b]$.

In these circumstances,

$$
\int_{a}^{b} u d x=\frac{1}{(2 N) !} \int_{a}^{b}(x-a)^{N}(b-x)^{N} f(x) d x,
$$

and one can prove the inequalities

$$
\begin{gathered}
\frac{1}{(b-a)^{N}} \int_{a}^{b} u d x>\sum_{i=1}^{n} \frac{1}{\left(a_{i}-a_{i-1}\right)^{N}} \int_{a_{t-1}}^{a_{i}} u_{i} d x \\
\left(\int_{a}^{b} u d x\right)^{1 /(N+1)}>\sum_{i=1}^{n}\left(\int_{a_{t-1}}^{a_{i}} u_{i} d x\right)^{1 /(N+1)},
\end{gathered}
$$


where $\left\{a_{0}, a_{1}, \ldots, a_{n}\right\}$ is any partition of $[a, b]$ and $u_{i}(i=1, \ldots, n)$ is the solution of the boundary value problem

$$
\left.\begin{array}{l}
\frac{d^{2 N}}{d x^{2 N}} u_{i}=(-1)^{N} f(x) \\
u_{i}=\frac{d u_{i}}{d x}=\cdots=\frac{d^{N-1}}{d x^{N-1}} u_{i}=0 \quad \text { at } x=a_{i-1} \text { and } x=a_{i} .
\end{array}\right\}
$$

The addition law

$$
\left(\int_{a}^{b} u d x\right)^{1 /(2 N+1)}=\sum_{i=1}^{n}\left(\int_{a_{i-1}}^{a_{i}} u_{i} d x\right)^{1 /(2 N+1)}
$$

is valid when $f$ is identically constant.

These conclusions would appear to have no obvious applications to mechanics if $N>2$.

Acknowledgment. I am indebted to an anonymous referee whose comments have enabled me to simplify and shorten the proofs of the main inequalities reported here.

\section{REFERENCES}

[1] W. A. Day, Positive deflections of elastic beams, Quart. Appl. Math. 44, 327-336 (1986)

[2] S. Friedland, On an inequality related to certain totally positive Green's functions, Quart. Appl. Math. 44, 337-338 (1986)

[3] P. Hartman, Ordinary Differential Equations, Wiley, New York, 1964 